\title{
To explore the clinical value of dual-energy virtual non-contrast CT scan for subarachnoid hemorrhage
}

\section{Bo Xiao \\ Department of Biomedical Engineering, Luzhou Medical College, Luzhou, Sichuan, 646000, P.R. China}

\section{Email address:}

2364061407@qq.com

\section{To cite this article:}

Bo Xiao. To Explore the Clinical Value of Dual-Energy Virtual Non-Contrast CT Scan for Subarachnoid Hemorrhage. Advances in Bioscience and Bioengineering. Vol. 1, No. 3, 2013, pp. 49-52. doi: 10.11648/j.abb.20130103.11

\begin{abstract}
Objectives: To study the clinical value of Dual-Energy Virtual Non-Contrast CT scan for subarachnoid hemorrhage. Methods: 30 patients of subarachnoid hemorrhage were selected, they received Dual-Source Conventional Non-Contrast CT scans and head angiography with Dual-Energy CT. Then Liver Virtual Non-Contrast program was used to reconstruct the images. After that the Conventional Non-Contrast images were taken as gold standard to evaluate the clinical value of Virtual Non-Contrast technology in the following aspects: Signal-Noise Ratio, Radiation Dose and lesion diagnosis. Results: The Signal-Noise Ratio of Virtual Non-Contrast CT scan was (4.21 \pm 0.5$)$, the Signal-Noise Ratio of Conventional Non-Contrast CT scan was $(10.39 \pm 0.71)$, the latter was significantly higher than the former. The Dose Length Product of Virtual Non-contrast CT scan was (121.30 \pm 3.8$)$, it was close to $1 / 6$ of the Dose Length Product (697.6 \pm 4.2$)$ of the Conventional Non-Contrast CT scan. As for the lesion area, the difference of the CT values between the Virtual Non-Contrast and the Conventional Non-Contrast CT scan was only $4 \sim 10 \mathrm{Hu}$. In addition, the Virtual Non-Contrast images' quality was graded by diagnostic teachers: there were 4 cases of 3 points, 23 cases of 2 points, 3 case of 1 point. Conclusions: Compared with the Conventional Non-Contrast images, there is greater noise in the Virtual Non-Contrast images, but the latter can meet the clinical diagnostic requirements and greatly reduce the radiation dose. Advances in knowledge: Virtual Non-Contrast technology was used for the diagnoses of subarachnoid hemorrhage, and the radiation dose was greatly reduced.
\end{abstract}

Keywords: Dual-Source CT, Virtual Non-Contrast CT Scan, Signal-Noise Ratio, Radiation Dose

\section{Introduction}

CT radiation has increasingly attracted more and more attention. The American Committee on radiation protection and measurements proposed protection against ionizing radiation should be possible to follow the principle of optimization of protection level [1], therefore, on the premise of meeting the diagnostic requirements, how to effectively reduce the radiation does become one of the focuses of our study. A series of methods, which can reduce the radiation dose of $\mathrm{CT}$ scan, have achieved effectiveness of varying degrees. These methods include reducing of $\mathrm{KV}$, $\mathrm{mAs}$, increasing the pitch and dose adjustment technology of real-time dynamic exposure, etc. Dual-Energy Virtual Non-Contrast (VNC) technology opened up a new way for the control of radiation does. Today VNC technology has been widely used in the scan of abdomen, in which the calculation is primarily based on the fat, water and other substances for virtual computing [2]. VNC technology is rarely used in the examinations of bleeding lesions in head, it is necessary to explore the methods of reducing radiation dose of head scan. In this paper, there were 30 patients of intracranial subarachnoid hemorrhage, they received Conventional Non-Contrast (CNC) CT scans for their heads and dual-energy angiography of their heads by using Simens dual-source CT, the images of different scan methods were compared, the images of the conventional non-contrast $\mathrm{CT}$ scans were taken as standard to evaluate the clinical value of VNC technology for subarachnoid hemorrhage in the following three aspects: the signal-noise ratio, the radiation dose, the lesion diagnosis, the feasibility and application value of VNC technology were studied. A conclusion was drawn: on the premise of ensuring that the image quality meets the diagnostic requirements, VNC technology can greatly reduce the radiation dose of the patient. 


\section{Methods and Materials}

\subsection{Case Selection}

From August 2012 to April 2013, 30 patients were randomly selected from West China Hospital of Sichuan University, 24 patients were male, 6 patients were female, male: female $=4: 1$, their ages were $40-76$ (their average age was 51.7). When the cases were selected, there were no special requirements for the age. All the patients suffered from subarachnoid hemorrhage and had received the operations.

\subsection{Instruments and Reagents}

Germany's Simens second-generation dual-source CT scanner was used. For these scans of the heads, the two sets of tubes were modulated to $80 \mathrm{KV}$ and $140 \mathrm{kv}$ respectively, the raw data were processed by the Simens post-processing software and were used to reconstruct the VNC images. The reagent iopamidol (the iopamidol was produced in Shanghai Brocco Sine Pharmaceutical Corp. Ltd., Shanghai, P.R.China) was selected in this study, iopamidol is non-ionic X-ray contrast reagent, its iodine content is high, it has a very good developing effect. In addition, it has low toxicity for the vessel walls and nerve tissue, it is stable and has less adverse reaction. Its dosage and uses: Cardiovascular Imaging: iopamidol $370[3] 1-1.2 \mathrm{ml} / \mathrm{kg}$. Enhanced CT scan: iopamidol 300-370,0.5-2 ml/kg.

\subsection{Examination Methods and Scan Parameters}

All the patients received convention CT scans for their heads and dual-energy head angiography. Before scanning, make sure that the patients had removed the metal objects, dentures and other jewelry from their bodies. Let the patient lie flat on the scanning bed, the head of the patient entered the CT gantry first, and the patient was told to keep his head stationary. Conventional non-contrast CT scan parameters: tube voltage $120 \mathrm{KV}$, tube current $340 \mathrm{mAs}$, step-scanning. Dual-source CT scan method: first the positioning images, which were from the skull base to the parietal skull, were scanned, then the patient received enhanced scan of the vessels in his head. High-pressure syringe needle was buried in the cubital vein of the patient. At the flow rate of $5.0 \mathrm{~mL} / \mathrm{s}$, binocular high-pressure syringe was used to inject non-ionic contrast agent iopamidol. In the internal carotid artery, region of interest was set to monitor the CT values, the threshold value was $100 \mathrm{Hu}$, the scanning direction was from the foot to the head. The scan parameters: Tube A voltage was $100 \mathrm{KV}$, its tube current was $104 \mathrm{~mA}$; Tube B voltage was Sn140KV, its tube current was $104 \mathrm{~mA}$. The pitch of the conventional non-contrast CT scan was 1.2, the pitch of the dual-energy CT scan was 0.6 .

\subsection{The Image Post-Processing and Data Collection}

The post-processing software of Siemens was used, the raw data of dual-source CT scan were imported in the post-processing software Liver VNC (Virtual Non-Contrast), the width and position of the window were adjusted and saved. Then the VNC images and the images of conventional non-contrast CT scan were simultaneously transferred to the 3D post-processing window of Siemens, the same bony landmarks were selected for the reconstruction of $5 \mathrm{~mm}$ thickness and 24 layers. The images of two sets of the same layer were transferred to the browser for data acquisition and comparisons.

Table 1. The number of lesions found in the Conventional Non-Contrast CT scan and the Virtual Non-Contrast CT scan

\begin{tabular}{lllll}
\hline The lesion display & $\begin{array}{c}\text { No fresh } \\
\text { bleeding }\end{array}$ & $\begin{array}{l}\text { Fresh } \\
\text { bleeding }\end{array}$ & Edema & $\begin{array}{l}\text { There are } \\
\text { metal } \\
\text { artifacts }\end{array}$ \\
\hline $\begin{array}{l}\text { Conventional Non-Contrast CT scan } \\
\text { Clear display }\end{array}$ & 29 & 1 & 12 & 8 \\
$\begin{array}{l}\text { Virtual Non-Contrast CT scan } \\
\text { Clear display }\end{array}$ & 28 & 1 & 10 & 5 \\
$\begin{array}{l}\text { Clear display with } \\
\text { low density }\end{array}$ & 1 & 0 & 0 & 12 \\
Fuzzy display & 0 & 0 & 2 & 1 \\
\hline
\end{tabular}

Table 2. The CT values and SNR of the Conventional Non-Contrast CT scan and the Virtual Non-Contrast CT scan (only one person's data)

\begin{tabular}{llll}
\hline Scan mode & Lesion area & $\begin{array}{l}\text { Normal brain } \\
\text { temporal lobe }\end{array}$ & Image SNR \\
\hline $\begin{array}{l}\text { The CT values of } \\
\text { the Virtual }\end{array}$ & 13.7 & 26.6 & 4.75 \\
$\begin{array}{l}\text { Non-contrast CT } \\
\text { scans }\end{array}$ & & \\
$\begin{array}{l}\text { The CT value of } \\
\text { the Conventional }\end{array}$ & 16.6 & 49.4 & 10.08 \\
$\begin{array}{l}\text { Non-contrast CT } \\
\text { scans }\end{array}$ & & \\
\hline
\end{tabular}

Table 3. The radiation dose of the Virtual Non-Contrast CT scan and the Conventional Non-Contrast CT scan

\begin{tabular}{lll}
\hline Scan mode & $\begin{array}{l}\text { CT Dose Index } \\
\text { volume (unit: } \mathbf{s})\end{array}$ & $\begin{array}{l}\text { The total amount of Dose } \\
\text { Length Product (unit: } \\
\text { mGy* cm) }\end{array}$ \\
\hline $\begin{array}{l}\text { Conventional } \\
\text { Non-contrast CT } \\
\text { scan }\end{array}$ & $48.4 \pm 10.81$ & $712.62 \pm 125.38$ \\
$\begin{array}{l}\text { Virtual } \\
\text { Non-contrast CT } \\
\text { scan }\end{array}$ & $6.525 \pm 0.195$ & $121.625 \pm 7.375$ \\
\hline
\end{tabular}

\subsection{The Images' Quality Assessment}

The CNC and VNC images were reviewed by two professor-level doctors in the Department of Radiology, and the VNC images' quality was graded in order to determine whether these images met the clinical requirements for subarachnoid hemorrhage. The grading criteria were as follows: 3 points: the VNC image could completely meet the requirements for reflecting the 
progress of the disease (Compared with the CNC image, the variations in the bleeding areas and the amount of bleeding were identical.); 2 points: the VNC image could basically meet the requirements for reflecting the progress of the disease(Compared with the $\mathrm{CNC}$ image, there were differences in the judgments of the scope and amount of bleeding); 1 point: the VNC image could not meet the requirements for reflecting the progress of the disease(Compared with the $\mathrm{CNC}$ image, the $\mathrm{VNC}$ image could not determine the progress of the disease). If the two researchers disagreed with each other, the third researcher had to participate in the discussion, and the three of them consulted to grade the image's quality.

\subsection{Statistical Processing}

By differential-square test and SPSS16.0 software processing, the data in Table 1, Table 2 and Table 3 was analyzed, then the value of $\mathrm{P}$ was obtained. $\mathrm{P}<0.05$, so it has statistical significance.

\section{Results}

\subsection{Assessment of the Image Quality and the Diagnostic Value of VNC CT Scan}

The obtained points of the VNC images' quality were as follows: 4 cases of 3 points, 23 cases of 2 points, and 3 cases $f 1$ point. Among all the 30 patients with subarachnoid hemorrhage, there were 29 patients who didn't have fresh bleeding, their images of CNC CT scans had normal densities, these images also had metal artifact interference but this did not affect the diagnosis. There were 28 patients who didn't have fresh bleeding in the VNC images, among them there were two patients' images with low peripheral densities, and the nature were not determined, too much noise could not be excluded from influencing these two images. However, in the VNC images, metal artifacts were effectively inhibited. Therefore, to some extend the diagnosis of VNC CT scans is superior to that of the CNC CT scan.

The patient of Fig. 1 and Fig. 2 was female, 47 years old. She suffered from spontaneous subarachnoid hemorrhage. In Fig. 1, the brain gray matter had a high contrast ratio , and the SNR(Signal-Noise Ratio) of the display was good, But the high density artifacts in the sphenopetrosal sclerotin and hemostatic clamp generated a lot of problems of the diagnosis, in which doctors could determine whether there were small scattered bleeding points surrounding the hemostatic clip. Fig. 2 was the head image of VNC CT scan, this image could basically show the structure, though the SNR and contrast ratio were much more worse than those of the CNC CT scan, the image of VNC CT scan could meet the diagnostic requirements. For metals and high-density bone, VNC CT scan is better than CNC CT scan.

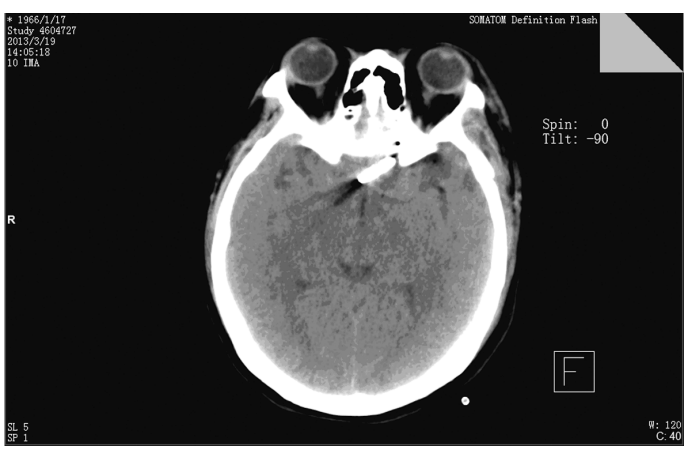

Figure 1 The CNC image of the patient's head

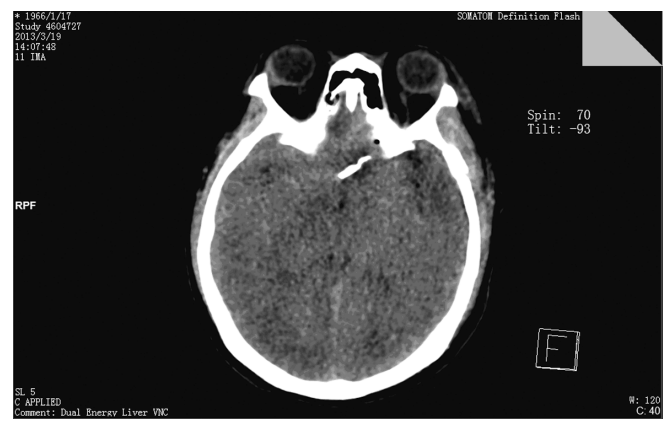

Figure 2 The VNC image of the patient's head

\subsection{Radiation Dose}

The values of CTDIvol (CT Dose Index volume) and DLP(Dose Length Product) of the CNC CT scan were higher than those of the VNC CT scan. Of course, the Effective Dose(ED) of the CNC CT scan was higher than that of the VNC CT scan. CTDIvol and DLP of the CNC CT scan was $(48.74 \pm 10.81)$ and $(712.62 \pm 125.38)$ respectively, CTDIvol and DLP of the VNC CT san was $(6.525 \pm 0.195)$ and $(121.625 \pm 7.375)$ respectively; The CTD value of the CNC CT scan is 7.47 times that of the VNC CT san, the DLP value is 5.88 times that of the VNC CT scan. Therefore, when the VNC CT scan is used to replace the $\mathrm{CNC} \mathrm{CT}$ scan, the radiation dose of the patient can be really reduced.

\subsection{Discussions and Conclusions}

Because there is a significant contrast of the CT densities between the vascular lesions of the head and the brain tissue \& the cerebrospinal fluid, the CNC CT scan is the preferred method for the diagnosis of these diseases. The major disease of the head vessels is rupture and hemorrhage of intracranial aneurysms. Multi-slice CTA has convenient, fast and non-invasive characteristics, and it can clearly display the neighboring region of the tumor, the positioning accuracy is very high, today multi-slice CTA has already become the preferred method of screening for intracranial aneurysmsy using DE-CT [4], the scan can be finished in one time, it effectively reduce the radiation dose, and it also has high reliability of determining the intracranial aneurysm, therefore DE-CT can be used as a routine method of screening for intracranial aneurysm. In clinical work, in general before CTA, the CNC CT scan is 
performed to further understand the bleeding lesions. If there is re-bleeding, then more times of CTA are performed, so the patients in a series of examinations are more likely to receive radiation dose. The use of dual-energy CTA can obtain virtual images directly, thereby it can replace the CNC CT scan, reduce the radiation dose of the patient and save the cost of the examination.

In the dual-energy $\mathrm{CT}$ scan, the $\mathrm{KV}$ values of the two tubes was set $80 \mathrm{KV}$ and $\mathrm{Sn} 140 \mathrm{KV}$, respectively. Therefore, for different $\mathrm{KV}$ values, the absorption coefficients of the same substance were different, and the CT values were also different. The CT values of $80 \mathrm{KV}$ and the $\mathrm{CT}$ values of Sn140KV were taken as abscissa and vertical coordinate, respectively, so the $\mathrm{CT}$ values changed with fixed slope in this graph. In the CT image, the CT values of the body tissue and the contrast agent changed in the form of different points. According to "Material Extraction Theory"[5], the points of the iodine injected in the body were removed, the obtained images were similar to the non-contrast images, they could be called images of virtual non-contrast CT scan. So the images of CTA and virtual non-contrast CT scan could be obtained at the same time, cerebral aneurysms and bleeding status could also be checked simultaneously.

By using the post-processing software, Dual-Energy Liver VNC of Siemens, the window width and position were adjusted to clearly display the brain tissue, and the iodine could be removed so as to achieve the VNC images [6]. Compared with the CNC CT scan, the radiation dose of VNC CT scan could be close to zero, this was because the images of VNC CT scan were obtained in the post-processing, and the patients did not essentially receive CT scans, therefore this was the biggest advantage of virtual non-contrast CT scan [7]. Since intracranial blood-vessels were generally located in the cerebral fissure and sulcus, the high-density contrast agent in the blood-vessels were adverse to observing the subarachnoid hemorrhage. In the observation with $\mathrm{VNC}$ images, you could obviously feel the noise increased significantly, the reason might be that the effective data were also removed in the process of filtering out the enhancer, plus the dose of the CT scan for the head was low in the first place, so the obtained data became less. Compared with the CNC CT scan, the SNRs of images in VNC CT scan were much lower. But in the fresh bleeding lesions, the blood and the normal brain tissue had a strong contrast in the VNC images [8], therefore, VNC CT scan could still achieve the diagnostic effects.

In the above study, the diagnostic accuracy of fresh bleeding was high. However for the 30 patients, most of them did not have fresh bleeding, but suffered from old bleeding or a small amount of bleeding, so the sensitivities of the VNC CT scan were not high, resulting in a lack of clarity, and this affected the final diagnoses of the patients. Therefore, virtual non-contrast CT scan has a high value for re-bleeding vessels of the head, at the same time it effectively reduces the radiation dose.

In summary, in the check of vascular lesions of the head, the CTA can be used, after the relative parameters are adjusted with the VNC technology, the images can be applied in the displays of the head. Though the VNC CT scan has a strong noise, it can meet the diagnostic requirements, and it has high accuracy for the vascular lesions of the head.

\section{References}

[1] Jiang Jie, Han Dan. The clinical application and progress of virtual non-contrast technology of dual-energy CT. Chinese Journal of Radiology. 2012,46(10):958 960

[2] Liang Yi, Dual-energy CT virtual non-contrasted images in patients suspected of having hepatic masses. Master's Degree thesis, Southern Medical University, 2010. Page: 25-37.

[3] Guo Xing, Ding Wei. Application of virtual non-enhanced images in evaluating subarachnoid hemorrhage by dual-energy Computed Tomography angiography. Acta Academiae Medicinae Sinicae. 2010,Vol.32 No.6:695 698

[4] Huang Wei, Xu Yiming. Dual-source virtual non-contrast CT of the head: a preliminary study. Chinese Journal of Radiology. 2011,Vol.45, No.3:229 233

[5] Zhan Libo, Qin Dongjing. The basic principle and characteristics of dual-source CT. Radiologic Practice. 2008;06: 55 59

[6] Lu Heqin, Zhu Guoying. To study the influencing factors of medical X-ray CT radiation dose. Shanghai Medical Imaging, 2008;02: 79 82

[7] Zhang longiia, Lu Guangming. The research progress of dual-energy imaging of dual-source CT. Journal of Clinical Radiology, 2009;11:22 26 